\title{
Spatially resolved photoluminescence in semiconductor nanostructures: A theoretical description
}

\author{
G. Pistone*, S. Savasta, O. Di Stefano, and R. Girlanda
}

Istituto Nazionale per la Fisica della Materia

and Dipartimento di Fisica della Materia e Tecnologie Fisiche Avanzate, Università di Messina,

Salita Sperone 31, 98166 Messina, Italy

\begin{abstract}
We present a microscopic quantum theory of spatially resolved photoluminescence in quantum wells with interface fluctuations that includes light quantization, acustic phonon scattering, and inhomogeneous sample-excitation and/or light-detection. The theory can model low-temperature photoluminescence and photoluminescence excitation experiments performed in illumination, collection or illumination-collection mode or diffusion experiments where the spatial positions of excitation and collection are scanned independently. Numerically calculated two-dimensional images agree with images from near-field photoluminescence experiments and put forward the potentials of the method for the understanding of near-field light emission from semiconductor quantum structures.
\end{abstract}

Near-field optical microscopy and spectroscopy, which uses optical interaction in the visible or nearinfrared range has demonstrated its ability to image optical fields and surface structures at a subwavelength scale. In particular the ability of this kind of optical microscopy and spectroscopy to identify the individual quantum constituents of semiconductor quantum structures has been widely demonstrated [1-4]. Among the most investigated quantum structures are quantum dot (QD) systems formed naturally by interface fluctuations in narrow quantum wells [5]. QD arrays have distributions in size and shape that lead to inhomogeneous spectral linewidth and averaged data. To avoid this problem these systems are mainly probed by means of techniques of high resolution spatially resolved spectroscopy and microscopy. In the last years measurements based on spatially-resolved photoluminescence (PL) provided direct information on the spatial and energy distribution of light emitting nanometric dots of semiconductor quantum structures $[1-4,6]$. Detailed simulations by Zimmermann and coworkers have clarified many aspects of the intrigued non-equilibrium dynamics giving rise to photoluminescence spectra in disordered quantum structures [7, 8]. However theoretical simulations of near-field imaging spectroscopy of semiconductor quantum structures mainly focus on calculations of local absorption [9-12]. In contrast as a matter of fact almost all experimental images are obtained from PL measurements. Here we present a microscopic quantum theory of spatially resolved photoluminescence in quantum structures that includes both light quantization (essential to describe spontaneous emission) and phonon scattering. The theory includes the description of spatially confined excitation (illumination mode) and/or detection (collection mode). This formulation thus permits to model spectroscopic imaging based on PL excitation spectroscopy in which the excitation and detection energies and spatial positions can all independently be scanned.

\footnotetext{
* Corresponding author: e-mail: pistone@unime.it, Phone: +390906765393, Fax: +39090391382
} 
The positive frequency components of the operator describing the signal that can be detected by a general near-field setup can be expressed as [13]

$$
\hat{S}_{t}^{+}=\hat{A}_{b g}^{+}+\hat{S}^{+},
$$

where $\hat{A}_{b g}^{+}$is the elastic background signal (largely uniform along the $x-y$ plane) proportional to the input electric-field operator, and $\hat{S}^{+}$is related to the sample polarization density operator $\hat{\boldsymbol{P}}^{+}(\boldsymbol{r})$,

$$
\hat{S}^{+}=\mathcal{A} \int \mathrm{d} \boldsymbol{r} \hat{\boldsymbol{P}}^{+}(\boldsymbol{r}) \cdot \boldsymbol{E}_{\text {out }}(\boldsymbol{r}),
$$

where $\mathcal{A}$ is a complex constant depending on the impedance of the material constituting the tip [13] and $\boldsymbol{E}_{\text {out }}(\boldsymbol{r})$ is the signal mode delivered by the tip. The interband polarization density operator is given by

$$
\hat{\boldsymbol{P}}^{+}(\boldsymbol{r})=\sum_{e h} \boldsymbol{\mu}_{e h} \hat{c}_{e}(\boldsymbol{r}) \hat{d}_{e}(\boldsymbol{r}) \text {. }
$$

Here, $e$ and $h$ are appropriate sets of quantum numbers which label the carrier states involved in the optical transition. Photoluminescence can be defined as the incoherent part of the emitted light intensity. The PL that can be measured by a photodetector after the collection setup (broadband detection) is proportional to $I=\left\langle\hat{S}^{-}, \hat{S}^{+}\right\rangle$, with $\langle\hat{A}, \hat{B}\rangle \equiv\langle\hat{A} \hat{B}\rangle-\langle\hat{A}\rangle\langle\hat{B}\rangle$. Analogously the steady-state spectrum of incoherent light emitted by the semiconductor quantum structure and detected by the scanning near field optical microscope (SNOM) setup can be expressed as

$$
I_{P L}\left(\omega_{\text {out }}\right)=\frac{1}{\pi} \int_{0}^{\infty} \mathrm{d} \tau\left\langle\hat{S}^{-}(0), \hat{S}^{+}(\tau)\right\rangle \mathrm{e}^{i \omega_{\text {out }} \tau} .
$$

The polarization density operator can be expressed in terms of exciton operators as,

$$
\hat{\boldsymbol{P}}^{+}(\boldsymbol{r})=\sum_{\alpha} \boldsymbol{\mu}_{e h} f(z) \Psi_{\alpha}^{e h}(\rho=0, \boldsymbol{R}) \hat{\boldsymbol{B}}_{\alpha} .
$$

The operator $\hat{\boldsymbol{B}}_{\alpha}^{\dagger}$ creates an exciton state (one electron-hole pair) $\hat{\boldsymbol{B}}_{\alpha}^{\dagger}|0\rangle \equiv\left|E_{1, \alpha}\right\rangle$ with energy $\omega_{1, \alpha}$. $\Psi_{\alpha}^{e h}(\rho, \boldsymbol{R})$ is the exciton wavefunction with $\rho$ indicating the relative in-plane electron-hole $(e h)$ coordinate and $\boldsymbol{R}$ describes the centre of mass motion, while $f(z)=u_{e}(z) u_{h}(z)$ is the product of the electron and hole envelope functions along the confinement direction (the growth axis). If the disorder induced broadening is small compared to the exciton binding energy, only the lowest bound state $1 s$ at the fundamental sublevel transition has to be considered and the exciton wave function can be factorized as follows [8]

$$
\Psi_{\alpha}^{e h}(\rho, \boldsymbol{R})=\phi_{1 s}(\rho) \psi_{\alpha}(\boldsymbol{R}) .
$$

At low excitation densities the operators $\hat{\boldsymbol{B}}_{\alpha}$ behave as Boson operators. Including only the exciton subspace, the Hamiltonian determining the dynamics of the semiconductor system is given by the following three contributions $H=H_{0}+H_{I}+H_{s}$, where the first term is the bare electronic Hamiltonian of the semiconductor system $H_{0}=\sum_{\alpha} \hbar \omega_{\alpha} \hat{B}_{\alpha}^{\dagger} \hat{B}_{\alpha}$. The interaction of the semiconductor with the light field (in the usual rotating wave approximation) can be written as

$$
H_{I}=-\int d^{3} r \hat{\boldsymbol{E}}^{-}(\boldsymbol{r}) \cdot \hat{\boldsymbol{P}}^{+}(\boldsymbol{r})+\text { H.c. } .
$$

We separate the field operator into a classical contribution $\boldsymbol{E}_{\text {in }}(\boldsymbol{r})$ describing the (possibly inhomogeneous) exciting field and into a fluctuating part $\hat{\mathcal{E}}^{-}(\boldsymbol{r})$ (the one determining the spontaneous emission) that can be expanded in terms of annihilation photon operators. Finally $H_{s}$ describes inelastic scattering due to the interaction of excitons with the phonon bath

$$
H_{s}=\sum_{\alpha, \beta, q} t_{\alpha, \beta}^{\boldsymbol{q}}\left(b_{\boldsymbol{q}}+b_{-\boldsymbol{q}}^{\dagger}\right) \hat{\boldsymbol{B}}_{\alpha}^{\dagger} \hat{\boldsymbol{B}}_{\beta} .
$$

These terms produce scattering between different exciton states and dephasing. For the lowest exciton states, scattering with acoustic phonons is in most cases the dominant process. $b_{\boldsymbol{q}}$ is the Bose annihilation operator for a phonon with wave vector $\boldsymbol{q}$. The scattering matrix elements depend on the lattice deformation potentials and the overlap between the exciton states. The explicit expression for $t_{\alpha, \beta}^{q}$ can be found elsewhere [8]. 
The kinetic equation for diagonal terms of the exciton density matrix $N_{\alpha}=\left\langle\hat{\boldsymbol{B}}_{\alpha}^{\dagger} \hat{\boldsymbol{B}}_{\alpha}\right\rangle$ can be derived starting from the Heisenberg equation of motion for the exciton operators under the influence of $H$. The main approximations are the neglect of possible coherent phonon states and of memory effects induced by the photon and phonon fields. Let us consider a possibly spatially resolved (illuminationmode) input light field of given frequency $\omega_{\text {in. }}$ The result is

$$
\partial_{t} N_{\alpha}=G_{\alpha}\left(\omega_{\text {in }}\right)+\sum_{\beta} \gamma_{\alpha \leftarrow \beta} N_{\beta}-2 \Gamma_{\alpha} N_{\alpha}
$$

where $\gamma_{\beta \leftarrow \alpha}$ are the resulting phonon-assisted scattering rates [8]. $2 \Gamma_{\alpha}=r_{\alpha}+\sum_{\beta} \gamma_{\beta \leftarrow \alpha}$ is the total outscattering rate, being $r_{\alpha}$ the rate for spontaneous emission, proportional to the exciton oscillator strength: $r_{\alpha}=r_{0}\left|\int d^{2} \boldsymbol{R} \psi_{\alpha}(\boldsymbol{R})\right|^{2}$. Here we assume that the tip-sample interaction does not alter the radiative decay rates. In this equation the generation term depends on the spatial overlap between the illuminating beam and the exciton wavefunctions corresponding to exciton levels resonant with the input light: $G_{\alpha}=r_{0}\left|o_{\alpha}^{\text {in }}\right|^{2} \mathcal{L}_{\alpha}\left(\omega_{\text {in }}\right)$ with $\pi \mathcal{L}_{\alpha}(\omega)=\Gamma /\left[\left(\omega-\omega_{\alpha}\right)^{2}+\Gamma^{2}\right]$ and $o_{\alpha}^{\text {in }}=\int d^{2} \boldsymbol{R} \tilde{E}_{\text {in }}(\boldsymbol{R}) \psi_{\alpha}(\boldsymbol{R})$, where $\tilde{E}_{\text {in }}(\boldsymbol{R})=\int E_{\text {in }}(\boldsymbol{r}) f(z) \mathrm{d} z$.

In the subsequent numerical calculations concerning the illumination-mode we will assume an input light field with a given Gaussian profile centered around the tip position: $\tilde{E}_{\text {in }}(\boldsymbol{R})=E_{\text {ing }}^{0} g(\boldsymbol{R}-\overline{\boldsymbol{R}})$. In this case the generation term becomes function of the beam position and shape (spatial resolution). We observe that also at steady-state Eq. (9) can give rise to highly non-equilibrium exciton densities. Non-equilibrium here arises from both spontaneous emission that prevents full thermalization and from the possible local excitation described by the generation terms.

Once the exciton densities have been derived, the frequency integrated PL can be readily obtained. Inserting Eq. (5) into Eq. (2), it results

$$
I\left(\overline{\boldsymbol{R}}_{\text {out }}\right)=r_{0} \sum_{\alpha}\left|o_{\alpha}^{\text {out }}\left(\overline{\boldsymbol{R}}_{\text {out }}\right)\right|^{2} N_{\alpha}
$$

where $o_{\alpha}^{\text {out }}$, analogously to $o_{\alpha}^{\text {in }}$, contains the overlap of the exciton wavefunctions with the signal mode $\tilde{E}_{\text {out }}(\boldsymbol{R})$ delivered by the tip centered at $\overline{\boldsymbol{R}}_{\text {out }}$ (collection mode).

According to the quantum regression theorem, $\left\langle\hat{S}^{-}(0) \hat{S}^{+}(\tau)\right\rangle$ has the same dynamics of $\left\langle\hat{S}^{+}(\tau)\right\rangle$ (proportional to the exciton operator), but with $\left\langle\hat{S}^{-}(0) \hat{S}^{+}(0)\right\rangle$ as initial condition. Following this procedure we obtain

$$
I_{P L}\left(\overline{\boldsymbol{R}}_{\text {out }}, \omega_{\text {out }}\right)=r_{0} \sum_{\alpha}\left|o_{\alpha}^{\text {out }}\left(\overline{\boldsymbol{R}}_{\text {out }}\right)\right|^{2} \mathcal{L}_{\alpha}\left(\omega_{\text {out }}\right) N_{\alpha}
$$

As a check of the above developed theoretical scheme we present some numerical results of spatially resolved PL. We consider a system of QDs arising from interface fluctuations of GaAs quantum wells. The effective disordered potential $V(\boldsymbol{r})$, used in our simulations, is modeled as a zero mean, Gauss distributed and spatially correlated process with amplitude $v_{0}=1 \mathrm{meV}$ and correlation length $\xi=20 \mathrm{~nm}$. Figure 1 displays two energy-integrated PL images $I(X, Y)$ obtained at two different tem-

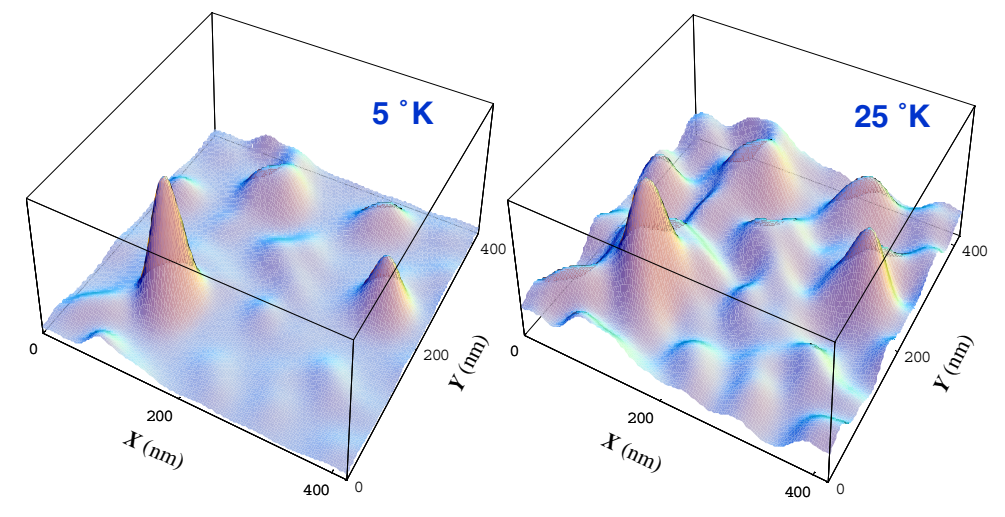

Fig. 1 Energy integrated spatially resolved photoluminescence in a disordered GaAs QW at two different temperatures. Parameters are given in the text. 

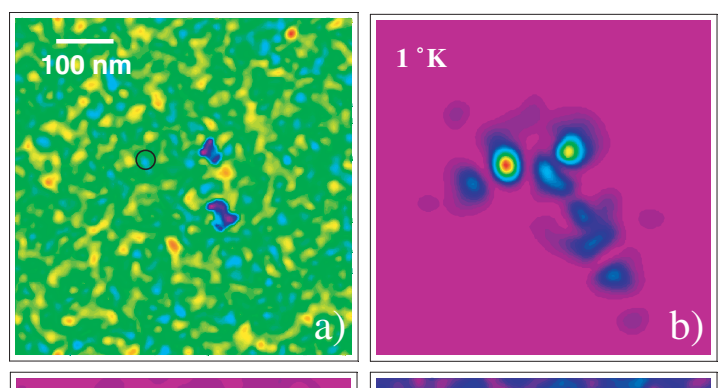

Fig. 2 a) Contour plot of the effective potential used for calculations shown in the panels $b-d$. The circle indicates the excitation location. b)-d) Energy integrated spatially resolved diffusion photoluminescence at different temperatures. Parameters are given in the text.
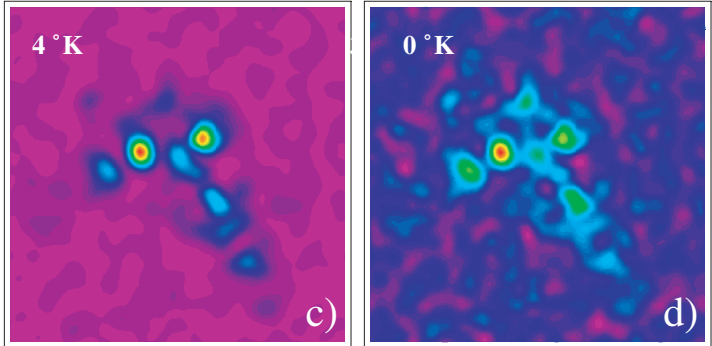

peratures ( $T=5$ and $25^{\circ} \mathrm{K}$ respectively). These spectra are obtained after uniform illumination of the sample at energy $\omega_{\text {in }}=2.6 \mathrm{meV}$ (the zero of energy is fixed at the energy of the $1 \mathrm{~s}$ exciton in absence of disorder) and collecting locally the emitted light (collection mode with spatial resolution $F W H M=47 \mathrm{~nm}$ ). When passing from $T=25^{\circ} \mathrm{K}$ to $T=5{ }^{\circ} \mathrm{K}$, we observe the transition from a broad and fairly continuous emission to an intense set of spatially localized emission peaks in correspondence of the potential minima. This behavior is also observed in numerous experiments.

Figure 2. displays a different kind of sample realization, with two naturally occuring dots plus minor fluctuations of shorter correlation length $\left(v_{0}=0.8 \mathrm{meV}\right.$ and $\xi=8 \mathrm{~nm}$ ). The two dots (see Fig. 2a) have a confinement energy of about $2 \mathrm{meV}$. The sample is locally excited at the location indicated by a black circle with a monochromatic beam $\left(\omega_{\text {in }}=-0.058 \mathrm{meV}, F W H M=30 \mathrm{~nm}\right)$. The energy-integrated images at three different temperatures $\left(T=1,4\right.$ and $30{ }^{\circ} \mathrm{K}$ respectively) are shown. These images are formed keeping fixed the illumination beam, while scanning the collection fiber all over the sample. At low temperature, light is mainly captured by the dot closer to the illumination zone. At increasing temperature, we observe how regions of the sample located away and away from the illuminated point becomes able to collect and hence to emit light.

\section{References}

[1] H. F. Hess et al., Science 264, 1740 (1994).

[2] D. Gammon et al., Appl. Phys. Lett. 67, 2391 (1995).

[3] D. Gammon et al., Phys. Rev. Lett. 76, 3005 (1996); Science 273, 87 (1996).

[4] Q. Wu et al., Phys. Rev. Lett. 83, 2652 (1999).

[5] A. Zrenner et al., Phys. Rev. Lett. 72, 3382 (1994).

[6] J. Hasen et al., Nature (London) 390, 54 (1997).

[7] R. Zimmermann and E. Runge, phys. stat. sol. (a) 164, 511 (1997).

[8] R. Zimmermann et al., Theory of resonant secondary emission: Rayleigh scattering versus luminescence, in: Quantum Coherence, Correlation and Decoherence in Semiconductor Nanostructures, edited by T. Takagahara (Elsevier Science, USA, 2003), p. 89-165.

[9] O. Mauritz et al., Phys. Rev. Lett. 82, 847 (1999).

[10] O. Di Stefano et al., Appl. Phys. Lett. 77, 2804 (2000).

[11] O. Di Stefano et al., J. Appl. Phys. 91, 2302 (2002).

[12] G. Pistone et al., Phys. Rev. B 67, 153305 (2003).

[13] J.-J. Greffet and R. Carminati, Prog. in Surf. Sci. 56, 133 (1997). 